\title{
Recent progress in staggered chiral perturbation theory
}

Jon Bailey, Weonjong Lee*, and Boram Yoon,

Lattice Gauge Theory Research Center, CTP, and FPRD,

Department of Physics and Astronomy,

Seoul National University, Seoul, 151-747, South Korea

E-mail: wlee@snu.ac.kr

\section{Hyung-Jin Kim,}

Physics Department, Brookhaven National Laboratory, Upton, NY11973, USA

E-mail: windy510@gmail.com

\section{SWME Collaboration}

We present a review on recent progress in staggered chiral perturbation theory (SChPT). In the last decade, the scope of the application of SChPT has been extended beyond the level of calibration into the region of prediction with high precision. SChPT becomes an essential tool to do the data analysis reliably for physical observables calculated using improved staggered fermions. Here, we focus on the following examples: pion spectrum, pion decay constants, $\varepsilon_{K}$, and $\pi-\pi$ scattering amplitudes. In each subject, we review the recent progress and future prospects.

The 7th International Workshop on Chiral Dynamics,

August 6 -10, 2012

Jefferson Lab, Newport News, Virginia, USA

\footnotetext{
*Speaker.
} 


\section{Introduction}

One of the most popular methods to put quarks on the lattice is the staggered fermion formalism. Staggered fermions and their improved versions preserve a part of the full chiral symmetry exactly and have a superior advantage of the cheapest cost to run on computers. However, staggered fermions are born with 4 tastes per flavor by construction. The disadvantage is that the SU(4) taste symmetry is broken at finite lattice spacing $(a>0)$, which is recovered only in the continuum limit $(a=0)$. In this paper, we review recent progress in staggered chiral perturbation theory (SChPT) which is designed to describe the chiral property of the physical observables calculated using improved staggered fermions.

\section{Staggered Chiral Perturbation Theory}

It is always possible to expand the lattice fermion action in powers of the lattice spacing $a$, which is quite useful to improve the action using the Symanzik program. In this expansion, the leading terms are the continuum QCD action, and the higher order terms contain the effect of lattice artifacts. SChPT is a kind of chiral perturbation theory to describe the chiral properties of physical observables calculated using the improved staggered fermions [1]. Hence, we map the lattice QCD action into a series in powers of $a^{2}$. The leading terms are the same as the continuum chiral perturbation theory. And the $a^{2}$ terms can map into the effective potential terms, which are of the same order as the leading terms in the power counting rules of $p^{2} \approx m_{\pi}^{2} \approx m_{q} \approx a^{2}$. Here, note that part of the power counting rules come directly from the numerical calculation of the pion multiplet spectrum on the lattice as in Refs. [2,3]. Hence, effectively we may say that SChPT corresponds to a dual expansion in $p^{2} \approx m_{\pi}^{2} \approx m_{q}$ and $a^{2}$. Since it is always possible to determine the complete set of operators at any given order in $a^{2}$ directly from the lattice symmetry group, we can say that SChPT incorporates all the tastes symmetry breaking effects into a dual series expansion order by order. As an accidental byproduct, we proved that the pion spectrum respects the $\mathrm{SO}(4)$ taste symmetry out of the full SU(4) symmetry at the leading order [1, 4], which is often called partial symmetry restoration. It turns out that the accidental $\mathrm{SO}(4)$ symmetry is a quite good approximation of the pion multiplet spectrum calculated using improved staggered fermions, as shown in Ref. [2,3].

The continuum QCD physics does not have taste degrees of freedom at all. Hence, for each sea quark loop, we need to reduce the number of tastes down to 1 . An efficient method to do this job is the rooting prescription. At finite lattice spacing where the taste symmetry is broken, the rooting leads to a non-trivial unphysical effect [5]. Here, we assume that this effect is taken care of properly in SChPT through the replica trick as suggested in Ref. [6], which is strongly supported by the numerical results in Ref. [7].

The SChPT Lagrangian

$$
\mathscr{L}_{\mathrm{LO}}=\frac{f^{2}}{8} \operatorname{Tr}\left(\partial_{\mu} \Sigma \partial_{\mu} \Sigma^{\dagger}\right)-\frac{1}{4} \mu f^{2} \operatorname{Tr}\left(M \Sigma+M \Sigma^{\dagger}\right)+\frac{2 m_{0}^{2}}{3}\left(U_{I}+D_{I}+S_{I}\right)^{2}+a^{2} \mathscr{V}
$$

where $M$ is a quark mass matrix $\operatorname{diag}\left(m_{u}, m_{d}, m_{s}\right) \otimes \xi_{I}$, and $\mathscr{V}$ is the effective potential which reflects the effect of taste symmetry breaking at $\mathscr{O}\left(a^{2}\right)$. This form of the SChPT Lagrangian was 
first proposed in Ref. [1] for a single flavor, later extended to multiple flavors in Ref. [4], and further extended to the next-to-leading order (NLO) in Ref. [8].

\section{Application of SChPT}

During the last decade, SChPT has become a standard tool to obtain the fitting functions for data analysis of lattice calculations done using improved staggered fermions. It has been applied to the pion spectrum, pion decay constants, $B_{K}$ and its BSM (beyond the standard model) corrections, decay constants of the heavy-light mesons, semileptonic form factors of the heavy-light mesons, and so on. Hence, it becomes impossible to cover the ingredients of interest over the whole subject in a few pages. Instead, we will select a few topics and focus on them to review the recent progress. Meanwhile, we also present the future prospects in these subjects.

\subsection{Pion masses and quark masses}

Pions made of staggered quarks can have flavor and taste structure $T_{f} \otimes \xi_{t}$, where $T_{f}$ will be Gell-Mann matrices in the case of SU(3) flavor symmetry and $\xi_{t}$ are the generators of the SU(4) taste symmetry. For simplicity of explanation, we choose the $\pi^{+}$flavor to fix the $T_{f}$ matrix. Then for a given $\pi^{+}$, we have 16 choices of $\xi_{t}$ : one $\left(\xi_{I}\right)$ belongs to a singlet irrep and the remaining 15 belong to an adjoint irrep of SU(4). The Goldstone pion corresponding to an exactly conserved axial current has the taste of $\xi_{5} \in \mathbf{1 5}$ adjoint irrep. The rest of the pions with taste not equal to $\xi_{5}$ are called non-Goldstone pions because the corresponding axial current is not conserved at finite lattice spacing.

At the leading order (LO), it was proved that the pion multiplet spectrum respects the $\mathrm{SO}(4)$ taste symmetry [1, 4], which turns out to be a very good symmetry. This indicates that there are 5 irreps in the pion multiplet spectrum. At NLO, it was proved that the $\mathrm{SO}(4)$ symmetry is broken down to $S W_{4}{ }^{1}[1,8]$. Hence, we have 8 irreps for the pion multiplet spectrum at NLO.

For the Goldstone pions, the NLO chiral logs were obtained in Ref. [4]. This calculation was extended to the non-Goldstone pions in Ref. [9]. At present, the SWME collaboration works on the mission to extend the NLO calculation to mixed actions such as HYP staggered valence quarks with asqtad sea quarks.

\subsection{Pion decay constants}

Calculation of pion decay constants on the lattice has multiple targets. The first is that we can determine $f_{\pi}, f_{K}, f_{K} / f_{\pi}$ ratio, which lead to a precise determination of $V_{u s}$. The second is that, combined with the pion spectrum, it can determine Gasser-Leutwyler low energy constants and chiral condensates.

There has been an attempt to explore the non-Goldstone pion sectors using the pion decay constants [10]. Recent progress in lattice calculation of pion decay constants are mainly focused on the Goldstone pion sector [7]. The NLO corrections in SChPT were obtained in Ref. [11]. This work has been recently extended to the non-Goldstone pion sectors in Ref. [12]. The SWME collaboration plans to extend this further to the mixed action case.

\footnotetext{
${ }^{1} S W_{4}$ is a finite subgroup of the $S O(4)$ group. The $S W_{4}$ is in the diagonal of the direct product of the taste and Euclidean $\mathrm{SO}(4)$ symmetries.
} 


\subsection{Indirect CP Violation and $B_{K}$}

The indirect CP violation observed in the neutral kaon system in nature is parametrized by $\varepsilon_{K}$, which is measured in experiment with extremely high precision as follows,

$$
\varepsilon_{K}=(2.228 \pm 0.011) \times 10^{-3} \times e^{i \phi_{\varepsilon}}, \quad \phi_{\varepsilon}=(43.52 \pm 0.05)^{\circ} .
$$

It is also possible to calculate $\varepsilon_{K}$ directly from the standard model. In the standard model, $\varepsilon_{K}$ can be expressed in terms of $B_{K}$ and $V_{c b}$ [13]. Here, $B_{K}$ is a highly non-perturbative parameter which can be determined reliably only using lattice QCD. In the case of $V_{c b}$, there are two independent methods to obtain from the experiment: one is the exclusive decay channel and the other is the inclusive decay channel. The exclusive method is simple and heavily relies on lattice QCD, whereas the inclusive method is very complicated and relies highly on QCD sum rules.

In Ref. [14], the NLO corrections to $B_{K}$ are obtained using the SU(3) SChPT. Here, they assumed that the external kaons have the same taste as the Goldstone pions of the staggered fermion formalism, which simplifies the technical aspects of calculation a lot. This work has been extended to the mixed action case and the $\mathrm{SU}(2)$ case in Ref. [15]. In addition, it turns out that the mixed pion (composed of valence quark and sea antiquark) contribution to $B_{K}$ completely cancels off between the numerator and the denominator at NLO, which makes $B_{K}$ a gold-plated observable in lattice QCD with a mixed action [15].

Results of the SU(2) SChPT in Ref. [15] provide the fitting functional form which is used for the lattice data analysis for $B_{K}$. Recent results for $B_{K}[16,17]$ indicate that there exists a substantial gap of $\approx 3 \sigma$ between the standard model prediction from lattice QCD and the experimental value of $\varepsilon_{K}$ in the exclusive $V_{c b}$ channel [13]. This gap may soon become a probe to identify physics beyond the standard model (BSM) by constraining models of new physics. There are, in general, 4 additional four-fermion operators which can come from the BSM physics. These four operators can be parametrized into four B-parameters: $B_{i}(i=2,3,4,5)$. Recently, the chiral behavior of the BSM B-parameters is presented at NLO in SChPT [18]. This work provides the fitting functional form which is used for the data analysis for the BSM B-parameters in Ref. [19].

\section{$3.4 \pi-\pi$ scattering and phase shift}

Let us consider $\pi-\pi$ scattering in the staggered fermion formalism. By construction, there are five non-degenerate channels of two pion states in a singlet irrep of the SU(4) taste symmetry instead of a single two pion state as in the case of Wilson-like fermions. In other words, the five two pion states are

$$
\pi(P)-\pi(P), \quad \pi(A)-\pi(A), \quad \pi(T)-\pi(T), \quad \pi(V)-\pi(V), \quad \pi(S)-\pi(S)
$$

Hence, we have to consider at least $5 \times 5 \mathrm{~S}$-matrix in staggered fermion formalism to calculate the $\pi-\pi$ scattering phase shift in the taste singlet channel. Recently, Hansen and Sharpe provided a prescription which allows us to handle the multi-channel scattering and decay problems in Ref. [20]. In this prescription, they assume that the multi-channel S-matrix is unitary.

In the staggered fermion formalism, we use the rooting method in the sea quark loops in vacuum polarization diagrams. Since the SU(4) taste symmetry is broken at finite lattice spacing, we know that the rooting makes it non-unitary to calculate the $\pi-\pi$ scattering diagrams using 
staggered fermions. This is bad news in itself. However, the good news is that we can calculate those terms which violate unitarity of the S-matrix order by order in SChPT. The strategy is that we fit the data to the functional form derived in SChPT and remove the unwanted part of unitarity violation terms by hand, and we use the remaining part, which corresponds to the unitary S-matrix. Then we can apply the Hansen-Sharpe formula to obtain the scattering phase shifts.

At present, the SWME collaboration is working on the first stage of how to dissect the unitarity violation part from the $\pi-\pi$ scattering amplitude.

\begin{tabular}{c|c|c|c|c}
\hline \hline physics & Goldstone & Non-Goldstone & mixed & numerical \\
\hline \hline$m_{\pi}^{2}$ & $\checkmark$ & $\checkmark$ & $\triangle$ & $\checkmark$ \\
\hline$f_{\pi}$ & $\checkmark$ & $\checkmark$ & $\triangle$ & $\checkmark$ \\
\hline$B_{K}$ & $\checkmark$ & $\times$ & $\checkmark$ & $\checkmark$ \\
\hline$\pi-\pi$ & \multicolumn{2}{|c|}{$\triangle$} & $\times$ & $\triangle$ \\
\hline$K \rightarrow \pi \pi$ & \multicolumn{2}{|c|}{$\times$} & $\times$ \\
\hline$V_{c b}$ & \multicolumn{2}{c}{$\times$} & $\checkmark$ \\
\hline \hline
\end{tabular}

Table 1: Current status of SChPT application. See text for key words.

\section{Summary and conclusion}

In Table 1, we present the current status in the application of SChPT to various physics subjects. Here, the $\checkmark$ symbol represents that the mission is completed, and $\times$ represents that no work has yet been done. The $\triangle$ symbol indicates that we are working on this subject now, and it will be done in near future.

\section{Acknowledgments}

We are grateful to Claude Bernard, Maarten Golterman, and Stephen Sharpe for private communications. The research of W. Lee is supported by the Creative Research Initiatives Program (2012-0000241) of the NRF grant funded by the Korean government (MEST). W. Lee would like to acknowledge the support from KISTI supercomputing center through the strategic support program for the supercomputing application research [No. KSC-2011-G2-06].

\section{References}

[1] W.-J. Lee and S. R. Sharpe, Partial flavor symmetry restoration for chiral staggered fermions, Phys.Rev. D60 (1999) 114503, [hep-lat/9905023].

[2] T. Bae, D. H. Adams, C. Jung, H.-J. Kim, J. Kim, et al., Taste symmetry breaking with HYP-smeared staggered fermions, Phys.Rev. D77 (2008) 094508, [arXiv: 0801.3000 ].

[3] MILC Collaboration Collaboration, C. Aubin et al., Light pseudoscalar decay constants, quark masses, and low energy constants from three-flavor lattice QCD, Phys.Rev. D70 (2004) 114501 , [hep-lat/0407028]. 
[4] C. Aubin and C. Bernard, Pion and kaon masses in staggered chiral perturbation theory, Phys.Rev. D68 (2003) 034014, [hep-lat/0304014].

[5] S. R. Sharpe, Rooted staggered fermions: Good, bad or ugly?, PoS LAT2006 (2006) 022, [hep-lat/0610094].

[6] C. Bernard, Staggered chiral perturbation theory and the fourth-root trick, Phys.Rev. D73 (2006) 114503, [hep-lat/0603011].

[7] A. Bazavov, D. Toussaint, C. Bernard, J. Laiho, C. DeTar, et al., Nonperturbative QCD simulations with 2+1 flavors of improved staggered quarks, Rev.Mod.Phys. 82 (2010) 1349-1417, [arXiv:0903.3598].

[8] S. R. Sharpe and R. S. Van de Water, Staggered chiral perturbation theory at next-to-leading order, Phys. Rev. D71 (2005) 114505, [hep-lat/ 0409018 ].

[9] SWME Collaboration Collaboration, J. A. Bailey, H.-J. Kim, and W. Lee, Taste non-Goldstone, flavor-charged pseudo-Goldstone boson masses in staggered chiral perturbation theory, arXiv:1112.2108.

[10] JLQCD Collaboration Collaboration, S. Aoki et al., Pion decay constant for the Kogut-Susskind quark action in quenched lattice QCD, Phys.Rev. D62 (2000) 094501, [hep-lat/9912007].

[11] C. Aubin and C. Bernard, Pseudoscalar decay constants in staggered chiral perturbation theory, Phys.Rev. D68 (2003) 074011, [hep-lat/ 0306026 ].

[12] J. A. Bailey, W. Lee, and B. Yoon, Taste non-Goldstone, flavor-charged pseudo-Goldstone boson decay constants in staggered chiral perturbation theory, arXiv:1212.5369.

[13] Y.-C. Jang and W. Lee, Current Status of Indirect CP Violation in Neutral Kaon System, PoS LATTICE2012 (2012) 269, [arXiv:1211. 0792].

[14] R. S. Van de Water and S. R. Sharpe, B(K) in staggered chiral perturbation theory, Phys.Rev. D73 (2006) 014003, [hep-lat/0507012].

[15] T. Bae, Y.-C. Jang, C. Jung, H.-J. Kim, J. Kim, et al., $B_{K}$ using HYP-smeared staggered fermions in $N_{f}=2+1$ unquenched QCD, Phys.Rev. D82 (2010) 114509, [arXiv:1008.5179].

[16] T. Bae, Y.-C. Jang, C. Jung, H.-J. Kim, J. Kim, et al., Kaon B-parameter from improved staggered fermions in $N_{f}=2+1$ QCD, Phys.Rev.Lett. 109 (2012) 041601, [arXiv:1111.5698].

[17] G. Colangelo, S. Durr, A. Juttner, L. Lellouch, H. Leutwyler, et al., Review of lattice results concerning low energy particle physics, Eur.Phys.J. C71 (2011) 1695, [arXiv: 1011.4408 ].

[18] J. A. Bailey, H.-J. Kim, W. Lee, and S. R. Sharpe, Kaon mixing matrix elements from beyond-the-Standard-Model operators in staggered chiral perturbation theory, Phys.Rev. D85 (2012) 074507, [arXiv:1202.1570].

[19] J. A. Bailey, T. Bae, Y.-C. Jang, H. Jeong, C. Jung, et al., Beyond the Standard Model corrections to $K^{0}-\bar{K}^{0}$ mixing, PoS LATTICE2012 (2012) 107, [arXiv: 1211.1101 ].

[20] M. T. Hansen and S. R. Sharpe, Multiple-channel generalization of Lellouch-Luscher formula, Phys.Rev. D86 (2012) 016007, [arXiv:1204.0826]. 